\title{
The B-Toolkit Demonstration
}

\author{
B-Core (UK) Ltd.
}

The Magdalen Centre, The Oxford Science Park, Oxford OX4 4GA, UK

The B-Toolkit is a suite of integrated programs which implement the B-Method for Software Development. The B-Method is a collection of mathematical based techniques which give a formal basis to those activities of Software Development that range from software specification, through design and integration, to code generation and into maintenance.

The B-Toolkit's components tools are implemented in the B Theory Language and is interpreted by the B-Tool. The B Theory language is a special purpose language for writing Software Engineering tools including interactive and automatic proof assistants and other tools where pattern matching, substitution and re-write mechanisms is used (e.g. translators, interpreters and generators).

Technical Description The B-Toolkit and the B-Method underwent eight years of research and development at British Petroleum, and its commercial development is done by B-Core(UK) Ltd. in Oxford. Many aspects of the B-Method have been devised by J.R.Abrial. The B-Toolkit covers many aspects of software engineering, including:

- Syntax and type-checking of specification documents as well as low level design documents, with comprehensive error reporting.

- Verification condition generation which generates the proof-obligations needed to guarantee specification consistency and correctness of refinement.

- Automatic \& interactive provers for discharging the verification conditions. Proof listing for visualising how the Automatic prover discharges proofobligations.

- Specification animation, enabling the specification to be 'run', validated and tested.

- A translator for translating low level design documents into C. Code generation from declarative descriptions, facilitated by a re-usable library of code modules.

- A Library of reusable code modules, which are all accessed and used according to their given specification.

- Rapid prototyping, facilitated by an interface generator, built on the reusable library.

- Automatic Production, Markup and Indexing of documentation of complete developments.

- Remaking facilities when a source file is altered; the scope of the remake is determined by the toolkit, and the system is rebuilt automatically to its former state, including code, documentation and proofs. 
- All tools are fully integrated and designed to run automatically. All tools are activated from a uniform portable comfortable Motif-based control panel which indicates the admissible commands. Help facilities are on-line.

The B-Method The B-Method is a mathematical method belonging to the school of thought known as the "model oriented" approach to software construction.

The notation, in particular the generalised substitution notation used by the B-Method is J.R.Abrial's extension to Dijkstra's calculus of guarded commands. The extension allows you to specify operations in terms of preconditions and postconditions. To the engineer, the formal notation looks like a simple pseudo programming notation. Also, there is no real distinction between the specification notation and the programming notation. The programming notation just appears as a subset of the specification notation.

The method and the notation have been designed together with the B-Toolkit that supports them, so that every aspect of the method has been validated by the genuine possibility of providing significant help to the engineer in using computer based tools to write, verify and maintain software.

Separate (object oriented) techniques within the method support the design of (large) specification and program construction. The complex dependencies within such big systems are automatically controlled by the tools.

The method is based on a series of embedded mathematical notations: the logical notation, the set notation (including the notation for relations, functions and sequences), the Natural Numbers, a substitution notation (predicate transformers), and the Abstract Machine Notation (AMN). The method and the notation is founded on set theory.

Industrial Usage In $1992 \mathrm{BP}$ International conducted an Alpha-test programme for the B-Toolkit. This Alpha-test programme involved 10 industrial sites in Europe. In January 1993, B-Core(UK) Limited started the Beta-test programme on 12 industrial and academic sites. In June 1994 the B-Toolkit became commercially available.

IBM UK has completed the development of a major component for CICS/ESA using the B-Toolkit for specification, proof, design and implementation.

In 1991 BP International developed a Refinery Graphics System for controlling flow of oil within a refinery. The system was 45,000 lines of $C$ of which 28,000 lines was developed using the B-Toolkit. The code generated by the B-Toolkit achieved high reliability and exceptional portability.

GEC Alsthom, Paris, have applied the B-Method to train control systems over the last 4 years. Matra Transport, Paris, has used the B-Toolkit extensively in specification of train control systems over the last 3 years. GEC-Marconi Aveonics is using the B-Toolkit for a pilot project developing embedded control systems. An industrial UK Users Trial Project led by Lloyds Register of Shipping, is providing continuous advice for further development of the B-Toolkit. 